\title{
Sätze über stetige Funktionen
}

In diesem Abschnitt wird bearbeitet:

Zwischenwertsatz, Satz von Maximum und Minimum, Bilder von Intervallen unter stetigen Funktionen

A59 Es sei $f$ eine Funktion $\mathbb{R} \rightarrow \mathbb{R}$ mit $f(1)=1$. Aus welcher Aussage kann man schließen, dass $f$ eine Nullstelle haben muss?

(1) $f$ ist stetig und $f(10)=-1$.

(2) $f$ ist streng monoton fallend und $f(10)=-1$.

(3) Aus jeder von beiden.

(4) Aus keiner von beiden.

A 60 Wenn eine Funktion $f:[a, b] \rightarrow \mathbb{R}$ kein Maximum hat, dann

(1) muss sie unstetig sein,

(2) muss sie unbeschränkt sein,

(3) beides,

(4) keines von beiden.

A61 Eine Funktion $f: \mathbb{R} \rightarrow \mathbb{R}$

(1) hat ein Maximum, wenn sie stetig ist,

(2) hat ein Maximum, wenn sie stetig und beschränkt ist,

(3) hat kein Maximum, wenn sie unstetig ist,

(4) keine dieser Aussagen ist richtig.

A 62 Es sei $f:[0,1] \cup[2,3] \rightarrow \mathbb{R}$ eine stetige Funktion. Was ist die stärkste Aussage, die man über ihre Bildmenge machen kann?

(1) Sie ist gleich $[f(0), f(3)]$.

(2) Sie ist ein Intervall.

(3) Sie ist gleich $[f(0), f(1)] \cup[f(2), f(3)]$.

(4) Sie ist die Vereinigung zweier Intervalle. 